\title{
Impaired Pain Processing Correlates with Cognitive Impairment in Parkinson's Disease
}

\author{
Akinori Okada ${ }^{1}$, Tomohiko Nakamura ${ }^{1}$, Junichiro Suzuki ${ }^{1}$, Masashi Suzuki ${ }^{1}$, \\ Masaaki Hirayama ${ }^{1,2}$, Masahisa Katsuno ${ }^{1}$ and Gen Sobue ${ }^{1,3}$
}

\begin{abstract}
Objective Pain and cognitive impairment are important clinical features in patients with Parkinson's disease (PD). Although pain processing is associated with the limbic system, which is also closely linked to the cognitive function, the association between pain and cognitive impairment in PD is still not well understood. The aim of the study was to investigate the association between pain processing and cognitive impairment in patients with PD.

Methods Forty-three patients with PD and 22 healthy subjects were studied. Pain-related somatosensory evoked potentials (SEPs) were generated using a thin needle electrode to stimulate epidermal A $\delta$ fibers. Cognitive impairment was evaluated using the Mini-Mental State Examination (MMSE), the Frontal Assessment Battery, and Japanese version of the Montreal Cognitive Assessment (MoCA-J), and their correlation with pain-related SEPs was investigated.

Results The N1/P1 amplitude was significantly lower in PD patients than the controls. N1/P1 peak-to-peak amplitudes correlated with the MMSE $(r=0.66, p<0.001)$ and MoCA-J scores $(r=0.38, p<0.01)$ in patients with PD. These amplitudes also strongly correlated with the domains of attention and memory in the MMSE (attention, $\mathrm{r}=0.52, \mathrm{p}<0.001$; memory, $\mathrm{r}=0.40, \mathrm{p}<0.01$ ) and MoCA-J (attention, $\mathrm{r}=0.45, \mathrm{p}<0.005$; memory, $\mathrm{r}=$ $0.48, \mathrm{p}<0.001$ ), but not in control subjects.

Conclusion A good correlation was observed between the decreased amplitudes of pain-related SEPs and an impairment of attention and memory in patients with PD. Our results suggest that pathological abnormalities of the pain pathway are significantly linked to cognitive impairment in PD.
\end{abstract}

Key words: Parkinson's disease, pain, cognitive impairments, attention, memory

(Intern Med 55: 3113-3118, 2016)

(DOI: 10.2169/internalmedicine.55.7067)

\section{Introduction}

Pain is an important and distressing symptom of Parkinson's disease (PD) (1). However, it is difficult to objectively assess pain, and challenging to determine how the mechanisms and pathophysiology of pain and PD are related. Painrelated somatosensory evoked potentials (SEPs) are considered to be a reliable method to objectively measure pain and have been used to identify possible single cerebral generators of pain-related signals $(2,3)$. Pain-related SEPs can be elicited by laser, heat, electric, or mechanical stimulation (4). Each stimulus used to activate a specific nociceptive receptor system in the skin evokes $A \delta$ fiber-mediated pain. Inui et al. recorded evoked potentials triggered by epidermal electrical stimulation using a thin needle electrode (5). They studied pain perception in healthy subjects by magnetoencephalography (MEG) and reported that the vertex biphasic SEP component approximately corresponded to activity of the medial temporal cortex. In patients with $\mathrm{PD}$, Tinazzi et al. recorded pain-related SEPs triggered by $\mathrm{CO}_{2}$ laser stimulation thought to produce musculoskeletal

\footnotetext{
${ }^{1}$ Department of Neurology, Nagoya University Graduate School of Medicine, Japan, ${ }^{2}$ Department of Pathophysiological Laboratory Science, Nagoya University Graduate School of Medicine, Japan and ${ }^{3}$ Nagoya University, Research Division of Dementia and Neurodegenerative Disease, Japan

Received for publication January 4, 2016; Accepted for publication March 25, 2016

Correspondence to Dr. Gen Sobue, sobueg@med.nagoya-u.ac.jp
} 
Table 1. Clinical Characteristics of Patients with PD and Control Subjects.

\begin{tabular}{lccc}
\hline & PD $(\mathrm{n}=43)$ & Control $(\mathrm{n}=22)$ & $\mathrm{p}$ value \\
\hline Gender (M:F) & $20: 23$ & $13: 9$ & 0.63 \\
Age (Y) & $66.5 \pm 5.8$ & $65.5 \pm 9.5$ & 0.76 \\
Disease duration (Y) & $4.5 \pm 3.3$ & & \\
UPDRS part III & $20.1 \pm 11.5$ & & \\
H \&Y & $2.1 \pm 1.1$ & & \\
L-dopa (mg/day) & $293 \pm 305$ & \\
\hline Values are expressed as mean (SD). UPDRS: United Parkinson's \\
Disease Rating Scales, H \& Y: Hoen and Yahr Scale
\end{tabular}

pain and reported that SEP amplitudes were significantly lower in patients with PD than in controls (6). In our previous study, we recorded pain-related SEPs triggered by electrical skin stimulation in patients with $\mathrm{PD}$, and pain-related SEPs were significantly lower in patients with PD than in controls (7).

Cognitive impairment is also a substantial non-motor symptom associated with PD, and it is present in approximately $45 \%$ of all PD patients (8). A study of cognitive impairment in patients with PD showed deficits in attention, memory, and working memory (9). Cognitive impairment is commonly associated with the limbic system and cholinergic system $(10,11)$. Both pain and cognitive impairment represent major obstacles in daily activities of the PD population (12), however, the association between cognitive impairment and pain processing is unclear. The aim of this study was to investigate the association between pain processing and cognitive impairment in patients with PD.

\section{Materials and Methods}

\section{Subjects}

Forty-three consecutive patients (20 men and 23 women) with PD and 22 healthy control subjects (13 men and 9 women) were studied. The characteristics of patients with PD and control subjects are shown in Table 1. Patients were recruited from the University of Nagoya University Hospital, Japan. Patients with PD fulfilled the diagnostic criteria for PD (13). Motor performance was assessed using the Hoehn and Yahr (H\&Y) scale and the Unified Parkinson's Disease Rating Scale (UPDRS) part III-motor examination. The levodopa equivalent daily dose was computed for each patient (14). The exclusion criteria included clinical findings of peripheral neuropathy, such as diabetic neuropathy, CharcotMarie-Tooth disease, or any other disease that could potentially cause sensory impairment. Patients taking analgesics or antidepressant treatments were also excluded. All patients with PD were examined during the on condition. None of the patients had taken any anti-cholinergic drugs, including medications for an over-active bladder.

The Ethics Committee of Nagoya University School of Medicine approved all aspects of this study. Written informed consent for participation was obtained from all sub- jects.

\section{Recording of SEPs by an intra-epidermal needle electrode}

We recorded pain-related SEPs using the methodology described previously and a custom-made needle electrode (Nihon-Koden, Tokyo, Japan) (7). The electrical stimulus was current evoked using a constant square wave pulse delivered at random intervals at $0.1 \mathrm{~Hz}$; the stimulus duration was $1.0 \mathrm{~ms}$. The current intensity was set to a level which produced a definite pain sensation in each subject described as painful. We stimulated the right side of the face approximately $3 \mathrm{~cm}$ below the infraorbital margin. A recording electrode was placed at the $\mathrm{Cz}$ (vertex) according to the 1020 international system. The reference electrode was applied to the right earlobe. We focused on the evoked potential responses recorded from the $\mathrm{Cz}$. In order to avoid habituation, in each stimulus condition, 10 responses with approximately 10 -s randomized stimulation intervals were collected and averaged in one trial. In addition, three trials were recorded over 2-min intervals. SEP components were identified according to latency and polarity and were labeled in accordance with a previous report (3). The peak-to-peak amplitude was measured for the vertex biphasic SEP component (N1/P1) after ensuring that our methodology was consistent and produced reproducible data.

\section{Neuropsychological assessment}

All patients with PD and control subjects were evaluated using the Mini-Mental State Examination (MMSE), the Japanese version of the Montreal Cognitive Assessment (MoCA-J) for general cognitive assessment, and the Frontal Assessment Battery (FAB) for frontal lobe cognitive function. We also evaluated the domains of the MMSE and MoCA-J with particular focus on attention, memory, orientation, executive functions, language abilities, and visuospatial abilities. The attention domain was assessed using digit span forwards, letter cancelation and number subtraction tasks. The memory domain was assessed using immediate and delayed word recall tasks. The orientation domain was assessed using temporal and spatial orientation tasks. The language domain was assessed using sentence repetition and animal naming tasks, and the visuospatial domain was assessed using cube copying and crock drawing tasks. The executive domain was assessed using the Trail Making Test B, verbal abstraction, digit span backwards and phonemic word fluency tasks. Cognitive tests were performed by other investigators who were blinded to the results of pain-related SEPs.

\section{Statistical analysis}

We calculated the mean and the standard deviation (SD) of all variables for all patients and control subjects. Unpaired t-tests or a variance analysis was used to compare differences between two independent groups. The chi-square test was used to compare sex distribution among groups. 
A

Control N1/P1 amplitude: $21.23 \mu \mathrm{V}$

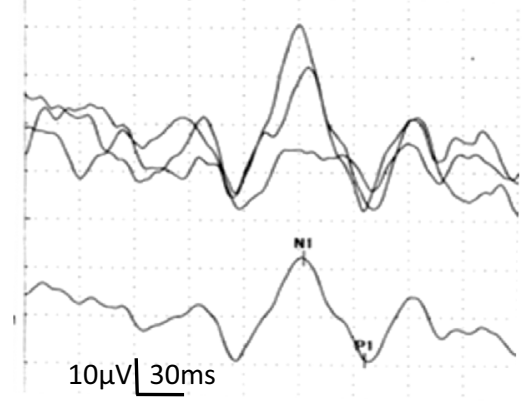

B

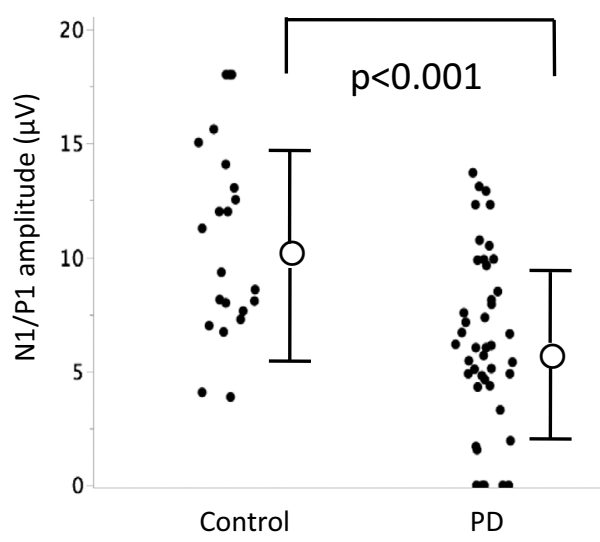

Figure 1. (A) Pain-related somatosensory evoked potentials (SEPs) of representative control subjects. (B) Comparison of the N1/P1 amplitude between patients with PD ( $=43$ ) and control subjects $(n=22)$. N1/P1 amplitudes were significantly lower in patients with PD than in control subjects. Symbols to the right of each group represent the mean \pm SDs.

Table 2. Results of Neuropsychological Tests.

\begin{tabular}{lccc}
\hline & PD $(\mathrm{n}=43)$ & Control $(\mathrm{n}=22)$ & $\mathrm{p}$ value \\
\hline MMSE & $28.5 \pm 1.4$ & $29.3 \pm 1.1$ & 0.10 \\
MoCA-J & $24.6 \pm 2.6$ & $25.3 \pm 2.7$ & 0.06 \\
FAB & $15.8 \pm 1.9$ & $15.7 \pm 2.1$ & 0.31 \\
\hline Values are & expressed as mean (SD). & MMSE: \\
Mini-Mental & State Examination, MoCA-J: & Japanese \\
version of the Montreal Cognitive Assessment, FAB: \\
Frontal Assessment Battery
\end{tabular}

Spearman's rank correlation was used to examine the relationship between variables. Statistical computing was performed with John's Macintosh Program (JMP) software program, version 11 , and a value of $\mathrm{p}<0.05$ was considered to denote statistical significance.

\section{Results}

\section{Pain-related SEP recordings}

Stimulus intensities were not significantly different between the patients with PD and the controls. Pain-related SEPs could not be evoked in five patients with PD. The amplitude of SEPs of these patients was included as zero and latency was excluded from the analysis. Fig. 1A shows waveforms that were evoked in representative control subjects. There were no significant differences in N1 or P1 latencies between patients with PD (N1, 185.7 $\pm 61.4 \mathrm{~ms}$; P1, $257.8 \pm 79.8 \mathrm{~ms})$ and controls $(\mathrm{N} 1,175.5 \pm 78.1 \mathrm{~ms} ; \mathrm{P} 1,245.5$ $\pm 65.0 \mathrm{~ms})$. However, the N1/P1 amplitudes were significantly lower in the patients with $\mathrm{PD}(6.3 \pm 3.8 \mu \mathrm{V})$ than in the controls $(10.8 \pm 4.3 \mu \mathrm{V})(\mathrm{p}<0.001)$ (Fig. 1B). There was no significant correlation between the N1/P1 amplitudes and age in both PD patients and controls. Additionally, there were no significant correlations between N1/P1 amplitudes and the disease duration, $\mathrm{H} \& \mathrm{Y}$ stage, UPDRS part III scores or levodopa equivalent daily dose in PD patients.

\section{Cognitive function scores and their correlation with N1/P1 amplitudes}

There were no significant differences between patients with PD and controls regarding the total scores of the MMSE, MoCA-J, or FAB (Table 2). In patients with PD, N1/P1 amplitudes positively correlated with the MMSE total scores $(r=0.66, p<0.001)$ and MoCA-J total scores $(r=0.38$, $\mathrm{p}<0.01$ ), but not FAB total scores. On the other hand, N1/P1 amplitudes did not correlate with the MMSE, MoCA-J, or FAB total scores in control subjects. Using a variance analysis, we observed significant differences in the slope regression line of pain-related SEPs, the MMSE and the MoCA-J between patients with PD and control subjects (Fig. 2). Furthermore, the reduction in N1/P1 amplitudes in the patients with PD showed a significant positive correlation with the domains of attention and memory in the MMSE and MoCA$\mathrm{J}$ scores, while the amplitude showed no significant correlation with other domains (Table 3). In the control subjects, however, the N1/P1 amplitudes did not correlate with the MMSE or MoCA-J scores.

\section{Comparison of pain-related SEPs between cogni- tively normal PD patients (PD-CN) and PD patients with mild cognitive impairment (PD-MCI)}

The PD patients were further classified into PD-CN and PD-MCI according to level II of the MDS commissioned Task Force (i.e., an impairment on neuropsychological tests may be demonstrated by performance approximately 1 standard deviation below appropriate controls) (15). The criteria for PD-CN were fulfilled in 30 patients and the criteria for PD-MCI were fulfilled in 13 patients. In addition, no signifi- 


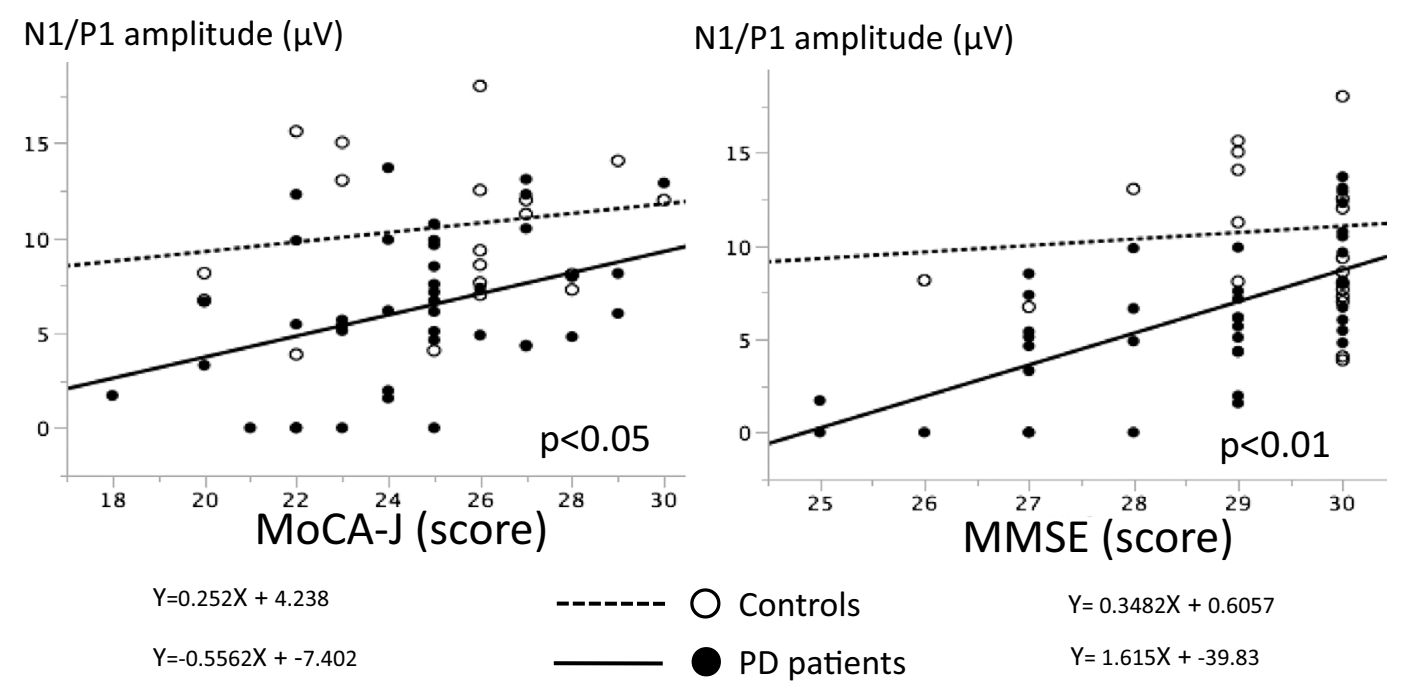

Figure 2. Correlation between the amplitude of pain-related potentials and MoCA-J and MMSE scores in patients with $P D(n=43)$ and control subjects $(n=22)$. The slope of the regression was significantly higher in patients with PD compared with controls.

Table 3. The Correlation between N1/P1 Amplitude and Cognitive Domain-compositive Scores in PD Patients and Control Subjects.

\begin{tabular}{ccccccc}
\hline & Attention & Memory & Orientation & Language & Visuospatial & Executive \\
\hline $\begin{array}{c}\text { PD }(\mathrm{n}=43) \\
\text { MMSE }\end{array}$ & & & & & & \\
r & 0.52 & 0.40 & 0.24 & 0.28 & 0.21 & \\
p value & $<.001$ & $<.01$ & 0.20 & 0.11 & 0.22 & \\
MoCA-J & & & & & & \\
r & 0.45 & 0.48 & 0.19 & 0.15 & 0.05 & 0.26 \\
p value & $<.005$ & $<.001$ & 0.14 & 0.46 & 0.74 & 0.10 \\
\hline Control (n=22) & & & & & & \\
MMSE & & & & & & \\
r & 0.35 & 0.30 & 0.40 & 0.18 & 0.15 & \\
p value & 0.12 & 0.16 & 0.08 & 0.55 & 0.70 & \\
MoCA-J & & & & & & \\
r & 0.08 & 0.02 & 0.35 & 0.40 & 0.15 & 0.23 \\
p value & 0.73 & 0.38 & 0.10 & 0.09 & 0.75 & 0.33 \\
\hline
\end{tabular}

Attention: digit span forwards, letter cancelation, number subtraction; memory: immediate and delayed word recall; orientation: temporal and spatial orientation; language: sentence repetition, animal naming;

visuospatial: cube copying, crock drawing; executive: Trail Making Test B, verbal abstraction, digit span backwards, phonemic word fluency. MMSE, Mini-Mental State Examination; MoCA-J, Japanese version of the Montreal Cognitive Assessment. p value of nonparametric Mann-Whitney-U test for independent samples.

cant differences in $\mathrm{N} 1$ or P1 latencies existed between PD$\mathrm{CN}(\mathrm{N} 1,183.3 \pm 31.7 \mathrm{~ms} ; \mathrm{P} 1,259.2 \pm 41.0 \mathrm{~ms})$ and PD-MCI patients $(\mathrm{N} 1,202.4 \pm 30.4 \mathrm{~ms}$; P1, 263.2 $\pm 40.8 \mathrm{~ms})(\mathrm{N} 1, \mathrm{p}=$ $0.10 ; \mathrm{P} 1, \mathrm{p}=0.12)$. However, there were significant differences in N1/P1 amplitudes between PD-CN $(8.1 \pm 4.4 \mu \mathrm{V})$ and PD-MCI patients $(5.1 \pm 5.4 \mu \mathrm{V})(\mathrm{p}<0.05)$. No significant differences in age, H\&Y stage, or the levodopa equivalent daily dose were observed between PD-CN and PD-MCI patients (data not shown).

\section{Discussion}

In the current study, we demonstrated that pain-related SEPs were closely related to an impaired cognitive function, particularly in terms of attention and memory, in patients with PD. Previous studies have revealed the pathological background of abnormal pain-related SEPs. For instance, in patients with central pain, pain-related SEP amplitudes are low and the reductions in amplitude are considered to indicate functional defects in the afferent pain pathway $(16,17)$. Tinazzi et al. showed a decrease in pain-related SEP amplitudes at the vertex by laser stimulating the skin of the limbs of patients with PD experiencing muscular pain, speculating that pain in PD is associated with additional changes in nociceptive processing (6). There have been many studies about pain stimuli. In healthy subjects, studies on pain using functional neuroimaging techniques have identified the thalamus, anterior cingulate cortex, somatosensory areas, insula, prefrontal cortex, amygdala, and other neighboring areas as pain processing regions $(18,19)$. Evidence derived 
from studies using MEG to monitor tactile and pain stimuli have indicated that anterior cingulate cortex activation is correlated with tactile and pain modalities (20). Moreover, a recent review of PD showed that the pathophysiological mechanisms of pain are associated with the hypofunction of the striatal dopaminergic system and pain-induced activation in the prefrontal and cingulate cortices $(21,22)$. These reported observations indicate that pain processing involves limbic structures, such as the anterior cingulate cortex and amygdala.

Pathologically, it is known that the limbic system, including the olfactory nucleus, amygdala, anterior cingulate cortex, and hippocampus, exhibits substantial pathological changes in patients with PD (23-27). We previously reported that patients with PD showed a correlation between scores on a smell function test and reductions in amplitudes of pain-related potentials, thus indicating the presence of a strong association between the limbic function and the ability to interpret sensory inputs (7). Therefore, we speculate that our results may show that common regions, such as the anterior cingulate cortex and hippocampus, are associated with pain processing and cognitive impairment in PD.

Attention dysfunction is generally considered to be associated with the reticular activating system or cholinergic system $(11,28)$. Previous studies on PD have reported that impairments of attention and memory are associated with cholinergic dysfunction $(29,30)$. It may be argued that since impairment of attention strongly influences memory function, the low scores in memory domains may be the result of impairment of attention. Therefore, there is a possibility that the attention and memory scores are concurrently low despite the absence of limbic system impairment. In addition, a transcranial magnetic stimulation (TMS) protocol, short-latency afferent inhibition (SAI), is known to be closely associated with cholinergic activity in CNS (31) and is reported to be attenuated in PD patients with dementia $(32,33)$. Previous reports demonstrated that at least some sensory systems are associated with cholinergic systems $(34,35)$. These observations suggest that impairment of cholinergic systems may be involved in pain perception in PD. Further studies to evaluate the relationships between SAI and pain-related SEP could reveal the relationship between the pain pathway and cholinergic dysfunction in PD. Cholinergic dysfunction is also reported to be closely associated with occipital dysfunction (36). Our results showed no correlation between pain-related SEPs and visuospatial dysfunction. This may be because the scores of visuospatial domains in the MMSE and MoCA-J are relatively small; thus, a small variance in the scores might lead to a poor correlation in our results.

There is one major limitation associated with this study. We did not classify the PD patients as having PD with pain or without pain. Further research on whether the amplitude of pain-related SEPs changes in PD with pain or without pain may clarify the association between pain-related SEPs and pain.
In conclusion, we herein showed an association between decreased pain-related SEP amplitudes and impaired attention and memory in patients with PD. Although the cholinergic systems play an important role in cognitive dysfunction of PD, our results suggest that pathological abnormalities of the limbic system also play a role in the pain pathway and cognitive dysfunction in patients with PD. Further research on whether the amplitude of pain-related SEPs changes following treatment of cognitive dysfunction using the advanced cognitive function test may clarify the association between the pain pathway and cognitive impairment more clearly in PD.

\section{The authors state that they have no Conflict of Interest (COI).}

\section{References}

1. Ford B, Louis ED, Greene P, Fahn S. Oral and genital pain syndromes in Parkinson's disease. Mov Disord 11: 421-426, 1996.

2. Kakigi R, Shibasaki H, Ikeda A. Pain-related somatosensory evoked potentials following $\mathrm{CO}_{2}$ laser stimulation in man. Electroencephalogr Clin Neurophysiol 74: 139-146, 1989.

3. Valeriani M, Rambaud L, Mauguière F. Scalp topography and dipolar source modelling of potentials evoked by $\mathrm{CO}_{2}$ laser stimulation of the hand. Electroencephalogr Clin Neurophysiol 100: 343353, 1996.

4. Handwerker HO, Kobal G. Psychophysiology of experimentally induced pain. Physiol Rev 73: 639-671, 1993.

5. Inui K, Tran TD, Hoshiyama M, Kakigi R. Preferential stimulation of A $\delta$ fibers by intra-epidermal needle electrode in humans. Pain 96: $247-252,2002$.

6. Tinazzi M, Recchia S, Simonetto S, et al. (2010) Muscular pain in Parkinson's disease and nociceptive processing assessed with $\mathrm{CO}_{2}$ laser-evoked potentials. Mov Disord 25: 213-220, 2010.

7. Hara T, Hirayama M, Mizutani Y, et al. Impaired pain processing in Parkinson's disease and its relative association with the sense of smell. Parkinsonism Relat Disord 19: 43-46, 2013.

8. Barone P, Antonini A, Colosimo C, et al; PRIAMO Study Group. The PRIAMO study: a multicenter assessment of nonmotor symptoms and their impact on quality of life in Parkinson's disease. Mov Disord 24: 1641-1649, 2009.

9. Pfeiffer HC, Løkkegaard A, Zoetmulder M, Friberg L, Werdelin L. Cognitive impairment in early-stage non-demented Parkinson's disease patients. Acta Neurol Scand 129: 307-318, 2014.

10. Sturm W, de Simone A, Krause BJ, et al. Functional anatomy of intrinsic alertness: evidence for a fronto-parietal-thalamicbrainstem network in the right hemisphere. Neuropsychologia 37: 797-805, 1999.

11. Nees F. The nicotinic cholinergic system function in the human brain. Neuropharmacology 96: 289-301, 2015.

12. Moriarty O, Mcguire BE, Finn DP. The effect of pain on cognitive function: a review of clinical and preclinical research. Prog Neurobiol 93: 385-404, 2011.

13. Hughes AJ, Daniel SE, Kilford L, Lees AJ. Accuracy of clinical diagnosis of idiopathic Parkinson's disease: a clinico-pathological study of 100 cases. J Neurol Neurosurg Psychiatry 55: 181-184, 1992.

14. Tomlinson CL, Stowe R, Patel S, Rick C, Gray R, Clarke CE. Systematic review of levodopa dose equivalency reporting in Parkinson's disease. Mov Disord 25: 2649-2653, 2010.

15. Litvan I, Goldman JG, Tröster AI, et al. Diagnotic criteria for mild cognitive impairment in Parkinson's disease: Movement Disorders Society Task Force guidline. Mov Disord 27: 349-356, 2012. 
16. Casey KL, Beydoun A, Boivie J, et al. Laser-evoked cerebral potentials and sensory function in patients with central pain. Pain 64: 485-491, 1996.

17. Garcia-Larrea L, Frot M, Valeriani M. Brain generators of laserevoked potentials: from dipoles to functional significance. Neurophysiol Clin 33: 279-292, 2003.

18. Hudson AJ. Pain perception and response: central nervous system mechanisms. Can J Neurol Sci 27: 2-16, 2000.

19. Rainville P, Duncan GH, Price DD, Carrier B, Bushnell MC. Pain affect encoded in human anterior cingulate but not somatosensory cortex. Science 277: 968-971, 1997.

20. Tanaka E, Inui K, Kida T, Miyazaki T, Takeshima Y, Kakigi R. A transition from unimodal to multimodal activations in four sensory modalities in humans: an electrophysiological study. BMC Neurosci 9: 116, 2008.

21. Brefel-Courbon C, Ory-Magne F, Thalamas C, Payoux P, Rascol O. Nociceptive brain activation in patients with neuropathic pain related to Parkinson's disease. Parkinsonism Relat Disord 19: 548552, 2013.

22. Conte A, Khan N, Defazio G, Rothwell JC, Berardelli A. Pathophysiology of somatosensory abnormalities in Parkinson disease. Nat Rev Neurol 9: 687-697, 2013.

23. Harding AJ, Stimson E, Henderson JM, Halliday GM. Clinical correlates of selective pathology in the amygdala of patients with Parkinson's disease. Brain 125: 2431-2445, 2002.

24. Braak H, Del Tredici K, Rub U, de Vos RA, Jansen Steur EN, Braak E. Staging of brain pathology related to sporadic Parkinson's disease. Neurobiol Aging 24: 197-211, 2003.

25. Sengoku R, Saito Y, Ikemura M, et al. Incidence and extent of Lewy body-related $\alpha$-synucleinopathy in aging human olfactory bulb. J Neuropathol Exp Neurol 67: 1072-1083, 2008.

26. Funabe S, Takao M, Saito Y, et al. Neuropathologic analysis of Lewy-related $\alpha$-synucleinopathy in olfactory mucosa. Neuropathology 33: 47-58, 2013.

27. Hall H, Reyes S, Landeck N, et al. Hippocampal Lewy pathology and cholinergic dysfunction are associated with dementia in Parkinson's disease. Brain 13: 2493-2508, 2014.

28. Garcia-Rill E, Kezunovic N, Hyde J, Simon C, Beck P, Urbano FJ. Coherence and frequency in the reticular activating system (RAS). Sleep Med Rev 17: 227-238, 2013.

29. Defazio G, Tinazzi M, Berardelli A. How pain arises in Parkinson's disease? Eur J Neurol 20: 1517-1523, 2013.

30. Hall H, Reyes S, Landeck N, et al. Hippocampal Lewy pathology and cholinergic dysfunction are associated with dementia in Parkinson's disease. Brain 137: 2493-2508, 2014.

31. Di Lazzaro V, Oliviero A, Tonali $P$, et al. Noninvasive in vivo assessment of cholinergic cortical circuits in $\mathrm{AD}$ using transcranial magnetic stimulation. Neurology 59: 392-397, 2002.

32. Celebi O, Temucin CM, Elibol B, Saka E. short latency afferent inhibition in Parkinson's disease patients with dementia. Mov Disord 27: 1052-1055, 2012.

33. Yarnall AJ, Rochester L, Baker MR, et al. Short latency afferent inhibition: a biomarker for mild cognitive impairment in Parkinson's disease? Mov Disord 28: 1285-1288, 2013.

34. Rothermel M, Carey RM, Puche A, Shipley MT, Wachowiak M. Cholinergis inputs from Basal forebrain add an excitatory bias to odor coding in the olfactory bulb. J Neurosci 34: 4654-4664, 2014.

35. Logemann HN, Böcker KB, Deschamps PK, Kemner C, Kenemans JL. The effect of the augumentation of cholinergic neurotransmission by nicotine on EEG indices of visuospatial attention. Behav Brain Res 260: 67-73, 2014.

36. Manganelli F, Vitale C, Santangelo G, et al. Functional involvement of central cholinergic circuits and visual hallucinations in Parkinson's disease. Brain 132: 2350-2359, 2009.

The Internal Medicine is an Open Access article distributed under the Creative Commons Attribution-NonCommercial-NoDerivatives 4.0 International License. To view the details of this license, please visit (https://creativecommons.org/licenses/ by-nc-nd/4.0/).

(C) 2016 The Japanese Society of Internal Medicine http://www.naika.or.jp/imonline/index.html 